Baseline MRI findings were not associated with re-injury (all, $\mathrm{p}>0.05)$.

Conclusions Presence of aponeurotic disruption and a runningrelated mechanism of injury resulted in longer return to play times for soleus injuries. Only clinical data, not MRI characteristics, were associated with risk of re-injury.

\section{CRITERIA-BASED RETURN TO SPORT DECISION-MAKING FOLLOWING LATERAL ANKLE SPRAIN INJURY: A RELEVANT PART OF THE PREVENTION - PERFORMANCE PARADOX FOR SECONDARY AND TERTIARY INJURY PREVENTION?}

\begin{abstract}
${ }^{1}$ Bruno Tassignon, ${ }^{1} J o$ Verschueren, 2,3 Eamonn Delahunt, ${ }^{4}$ Michelle Smith, ${ }^{4}$ Bill Vicenzino, ${ }^{5}$ Evert Verhagen, ${ }^{1,6}$ Romain Meeusen. ${ }^{1}$ Human Physiology and Sports Physiotherapy Research Group, Faculty of Physical Education and Physiotherapy, Vrije Universiteit Brussel, Brussels, Belgium; ' ${ }^{2}$ School of Public Health, Physiotherapy and Sports Science, University College Dublin, Dublin, Ireland; ${ }^{3}$ Institute for Sport and Health, University College Dublin, Dublin, Ireland; ${ }^{4}$ Division of Physiotherapy, School of Health and Rehabilitation Sciences, The University of Queensland, St Lucia, Queensland, Australia; ${ }^{5}$ Amsterdam Collaboration on Health and Safety in Sports, Department of Public and Occupational Health, Amsterdam Movement Sciences, Amsterdam UMC, Vrije Universiteit Amsterdam, Amsterdam, Netherlands; ${ }^{6}$ Strategic Research Program Exercise and the Brain in Health and Disease: the added value of Human-Centered Robotics, Vrije Universiteit Brussel, Brussels, Belgium
\end{abstract}

\subsection{6/bjsports-2021-IOC.34}

Background Lateral ankle sprain (LAS) injury is one of the most frequently incurred musculoskeletal injuries and shows high recurrence rates in individuals participating in sports. This increased re-injury risk is hypothesised to be mainly caused by the persistence of sensorimotor impairments and premature return to sport (RTS) clearance. Therefore, it is indicated to develop and implement evidence-based criteria to guide RTS decisions for LAS patients in order to minimize reinjury risk and maximise performance.

Objective To identify prospective studies that used a criteriabased RTS decision-making process for LAS patients.

Design Systematic review.

Setting Secondary and tertiary injury prevention.

Patients LAS patients.

Main Outcome Measurements Studies were included if they prospectively applied a criteria-based RTS decision-making process for LAS patients.

Results No relevant studies could be identified. However, we retrieved 47 relevant questionnaires, as well as 45 clinical and functional assessments commonly used in studies during the search process.

Conclusions There are currently no published evidence-based criteria to inform RTS decisions for LAS patients. It seems that RTS decisions following LAS injury are still generally time-based. We propose seven variable categories that could be used to develop a criteria-based RTS decision paradigm. These categories were generated in accordance with our results (i.e. questionnaires and tests) and the latest scientific insights. In summary, RTS decisions should acknowledge both preventive and performance criteria. Finding the right balance between these two divergent purposes within the rehabilitation process, is essential in order to minimize the athlete's re-injury risk and maximise performance. It is thus of paramount importance that all relevant stakeholders (e.g. athlete, medical staff, coaching, staff) take part in the RTS decision-making process. Future research should aim to reach consensus on these variables and apply them to actual RTS decisions within prospective study designs to evaluate re-injury risk and successful return to performance.

\section{HEAVY SHOULDER STRENGTHENING EXERCISES IN PATIENTS WITH HYPERMOBILITY AND LONG-LASTING SHOULDER SYMPTOMS: A FEASIBILITY STUDY}

${ }^{1}$ Behnam Liaghat, ${ }^{1,2}$ Søren T Skou, ${ }^{3}$ Uffe Jørgensen, ${ }^{4}$ Jens Sondergaard, 5,6 Karen Søgaard, ${ }^{1}$ Birgit Juul-Kristensen. 'Research Unit for Musculoskeletal Function and Physiotherapy, Department of Sports Science and Clinical Biomechanics, University of Southern Denmark, Odense, Denmark; ${ }^{2}$ Department of Physiotherapy and Occupational Therapy, NæstvedSlagelse-Ringsted Hospitals, Næstved, Slagelse, Ringsted, Denmark; ${ }^{3}$ Orthopedic Research Unit, Odense University Hospital, University of Southern Denmark, Odense, Denmark; ${ }^{4}$ Research Unit of General Practice, Faculty of Health Science, University of Southern Denmark, Odense, Denmark; ${ }^{5}$ Research Unit of Physical Activity and Health in Work Life, Department of Sports Science and Clinical Biomechanics, University of Southern Denmark, Odense, Denmark; ${ }^{6}$ Department of Clinical Research, University of Southern Denmark, Odense, Denmark

\subsection{6/bjsports-2021-IOC.35}

Background Patients with hypermobility spectrum disorder (HSD) are in great risk of experiencing shoulder symptoms, but evidence for exercise as treatment is sparse.

Objective To evaluate the feasibility of heavy shoulder strengthening exercise in patients with HSD and long-lasting shoulder symptoms.

Design Feasibility study.

Setting Primary care.

Patients (or Participants) Twelve patients (39.3 \pm 13.9 years) with HSD and shoulder instability and/or pain $>3$ months.

Interventions (or Assessment of Risk Factors) 16-week progressive heavy shoulder strengthening programme 3 times/ week using exercises targeting scapular and rotator cuff muscles.

Main Outcome Measurements Pre-defined progression criteria included recruitment rate (acceptable: 6 patients/month), test duration (acceptable: <120 min), patient retention (acceptable: $>80 \%$ complete intervention), training adherence (acceptable: $>75 \%$ adhere to $>36$ training sessions), adverse events (acceptable: minor events with no patients discontinuing the study), besides patient and physiotherapist feedback. Treatment outcomes were assessed using patient-reported health parameters, such as the Western Ontario Shoulder Instability Index (WOSI (0-2100, better to worse)), outcomes on pain, fatigue and kinesiophobia, besides isometric shoulder strength and clinical tests (shoulder instability, hypermobility and proprioception).

Results Recruitment rate was 5.6/month, assessment duration (mean \pm SD) $105 \pm 9 \mathrm{~min}$, retention $100 \%$, adherence $83 \%$, and four patients experienced short-lasting soreness/pain. Patient feedback was positive, and physiotherapists found the intervention relevant and applicable to the patient-group. WOSI total score improved by $51 \%$ (mean \pm SD, points: baseline 1037 \pm 215 ; follow-up 509 \pm 365 ; mean change (95\% CI), 528 $(318 ; 738))$, and patients reported reduced pain, fatigue and kinesiophobia. Shoulder strength measurements improved by 28-31\% (mean change (95\% CI), Nm/kg: scaption $0.51(0.23$; $0.78)$; internal rotation $1.32(0.70 ; 1.95)$; and external 
rotation $0.89(0.37 ; 1.40))$. Clinical tests indicated increased shoulder stiffness.

Conclusions The shoulder strengthening programme was feasible in patients with HSD and long-lasting shoulder symptoms. A future randomised controlled trial, with an improved recruitment strategy, will demonstrate whether the exercise programme is effective in improving symptoms in this patient group.

\section{SONOGRAPHIC TENDON ABNORMALITIES CAN PREDICT SYMPTOMATIC TENDINOPATHY IN MARATHON RUNNERS}

Sarah Eby, Masaru Teramoto, Rudi Zurbuchen, Keith Cummings, Joy English, Daniel M Cushman. University of Utah, Salt Lake City, USA

\subsection{6/bjsports-2021-I0C.36}

Background Tendinopathies of the patellar and Achilles tendons are common injuries among athletes and the general population. These injuries are costly, time-intensive to treat, and significantly limit activity. Predicting and eventually preventing these injuries is invaluable.

Objective Evaluate the relationship between sonographic tendon abnormalities and development of future injury in community marathon runners.

Design Prospective longitudinal cohort study. A blinded reviewer rated pre-race ultrasound images for tendon abnormalities, including thickening, hypoechogenicity, intratendinous delamination, or calcifications.

Setting 2019 Salt Lake City Marathon

Participants Marathon or half-marathon runners with no knee or ankle symptoms. 166 were screened; 138 completed the study $(36.2 \pm 12.0$ years of age, male: $n=68)$.

Assessment of Risk Factors Ultrasound evaluation of bilateral Achilles and patellar tendons immediately prior to the race and longitudinal surveys of subsequent injury development immediately following the race, and 1 and 3 months postrace.

Main Outcome Measurements Patellar or Achilles injury development. Statistical analyses included calculating relative risk (RR) with a 95\% confidence interval (CI) and hazard ratio (HR) with a 95\% CI from the Cox proportional hazards $(\mathrm{PH})$ model.

Results Sonographic abnormalities were found among $12.3 \%$ (left Achilles) and 26.8\% (right patellar) of runners. Runners with sonographic abnormalities were 2 times $(\mathrm{RR}=2.20$, $95 \% \mathrm{CI}=1.15,4.22, p=.040)$ more likely to develop pain in the Achilles tendon within three months compared to those with normal ultrasounds. The Cox $\mathrm{PH}$ model indicated that sonographic abnormalities were associated with 2 times (HR $=2.35,95 \% \mathrm{CI}=1.10,4.99, p=.027$ ) higher risk of developing post-race pain in the Achilles tendon after adjusting for runners' demographics and training regimens. No significant findings were observed between sonographic abnormality and post-race pain in the patellar tendon for the current observation period.

Conclusions Pre-race sonographic Achilles tendon abnormalities are associated with development of Achilles tendon pain within 3 months. Subsequent injury development, including at 6,9 , and 12-months post-race is pending.

\section{SHOULDER ROTATION STRENGTH CHANGES FROM PRESEASON TO MIDSEASON: A COHORT STUDY OF 292 YOUTH ELITE HANDBALL PLAYERS WITHOUT SHOULDER PROBLEMS}

${ }^{1}$ Behnam Liaghat, ${ }^{2}$ Jesper Bencke, ${ }^{3}$ Mette Kreutzfeldt Zebis, ${ }^{4}$ Henrik Sørensen, ${ }^{5}$ Grethe Myklebust, ${ }^{6}$ Niels Wedderkopp, ${ }^{7}$ Martin Lind, ${ }^{1}$ Merete Møller. ${ }^{1}$ Research Unit for Musculoskeletal Function and Physiotherapy, Department of Sports Science and Clinical Biomechanics, University of Southern Denmark, Odense, Denmark; ${ }^{2}$ Human Movement Analysis Laboratory, Copenhagen University Hospital, Amager-Hvidovre, Copenhagen, Denmark; ${ }^{3}$ Department of Physiotherapy, Faculty of Health and Technology, University College Copenhagen, Copenhagen, Denmark; ${ }^{4}$ Department of Public Health, Section for Sport Science, Aarhus University, Aarhus, Denmark; ${ }^{5}$ Department of Sports Medicine, Oslo Sports Trauma Research Center, Norwegian School of Sport Sciences, Oslo, Norway; ${ }^{6}$ Department of Regional Health Research, University of Southern Denmark, Odense, Denmark; ' Div. of Sportstraumatology, Aarhus University Hospital, Aarhus, Denmark

\subsection{6/bjsports-2021-IOC.37}

Background Shoulder rotation strength deficit measured at one time-point during preseason has been investigated as a risk factor for shoulder problems in sports with conflicting results. However, athletes face changes in physical demands and accumulative training exposure during a season, which likely influence their rotation strength over this period.

Objective We aimed to investigate if shoulder rotation strength changes during a competitive season in a cohort of youth elite handball players.

Design Prospective cohort study.

Setting Danish youth elite handball clubs.

Patients (or Participants) Players ( $\mathrm{n}=292,45 \%$ girls, 14-18 years) without shoulder problems were assessed at preseason and at midseason.

Interventions (or Assessment of Risk Factors) We measured isometric internal rotation (IR) and external rotation (ER) strength in supine with the shoulder abducted $90^{\circ}$ in neutral rotation and $30^{\circ}$ of IR measured using hand-held dynamometry, and the corresponding ER/IR ratios.

Main Outcome Measurements Changes in shoulder strength and ER/IR strength ratios.

Results From preseason to midseason, ER/IR mean ratios were higher in neutral rotation (boys difference 0.02 , 95\% CI 0.00 to 0.05 ; girls difference 0.05 , 95\% CI 0.010 .09 ) and in $30^{\circ}$ IR (boys difference 0.15 , 95\% CI 0.11 to 0.19 ; girls difference 0.12 , 95\% CI $0.07-0.17$ ) due to an increase in ER strength and a decrease in IR strength.

Conclusions The present finding supports that shoulder strength ratios change between two time points during a competitive season. Clinicians and researchers should be aware that strength in youth elite handball players may have a normal variation over the course of the season. This warrants ongoing monitoring and should be considered when normative reference values are compared, and when measuring the effect of targeted exercise programmes. Studies investigating if shoulder strength is a causal factor for shoulder problems should consider including the strength variable as a time-varying covariate. 